\title{
THE APOLLONIAN METRIC: LIMITS OF THE COMPARISON AND BILIPSCHITZ PROPERTIES
}

\author{
PETER A. HÄSTÖ
}

Received 12 July 2003

The Apollonian metric is a generalization of the hyperbolic metric. It is defined in arbitrary domains in $\mathbb{R}^{n}$. In this paper, we derive optimal comparison results between this metric and the $j_{G}$ metric in a large class of domains. These results allow us to prove that Euclidean bilipschitz mappings have small Apollonian bilipschitz constants in a domain $G$ if and only if $G$ is a ball or half-space.

\section{Introduction}

The Apollonian metric is a generalization of the hyperbolic metric introduced by Beardon [2]. It is defined in arbitrary domains in $\mathbb{R}^{n}$ and is Möbius invariant. Another advantage over the well-known quasihyperbolic metric [8] is that it is simpler to evaluate. On the downside, points cannot generally be connected by geodesics of the Apollonian metric. This paper is the last in a series of four papers on the Apollonian metric, the first three being $[9,10,11]$. Other authors who have approached this metric from the same perspective, providing the incentive for this investigation, are Rhodes [13], Seittenranta [14], Gehring and Hag [5, 6], and Ibragimov [12]. As becoming of a concluding paper, we will return here to the beginning and take a new look at the comparison and bilipschitz properties considered in [10]. Using results from [9], we are able to answer a question posed to the author by M. Vuorinen, which led to the start of this investigation, namely: under what circumstances are Euclidean bilipschitz with small distortion also Apollonian bilipschitz mappings with small distortion? This question can be seen as a step towards answering the question asked in [2] by Beardon about the isometries of the Apollonian metric, since the comparison condition has previously been shown to imply quite some regularity of the Apollonian metric (cf., e.g., the proof of [10, Theorem 1.6]). 
1142 Limits of the comparison and bilipschitz properties

We start by stating the main results and, at the same time, we sketch the structure of the rest of the paper. The notation used conforms largely to that of $[1,17]$, the reader can consult Section 2.

We will be considering domains (open connected nonempty sets) $G$ in the Möbius space $\overline{\mathbb{R}^{n}}:=\mathbb{R}^{n} \cup\{\infty\}$. The Apollonian metric for $x, y \in G \varsubsetneqq \mathbb{R}^{n}$ is defined by

$$
\alpha_{G}(x, y):=\sup _{a, b \in \partial G} \log \frac{|a-x|}{|a-y|} \frac{|b-y|}{|b-x|}
$$

(with the understanding that if $a=\infty$, then we set $|a-x| /|a-y|=1$ and similarly for $b$; see also Section 2.2). It is in fact a metric if and only if the complement of $G$ is not contained in a hyperplane, as was noted in [2, Theorem 1.1].

To define the comparison property, we need the $j_{G}$ metric, which is a modification from [16] of a metric introduced in [7]. This metric is defined for $x, y \in G \varsubsetneqq \mathbb{R}^{n}$ by

$$
j_{G}(x, y):=\log \left(1+\frac{|x-y|}{\min \{d(x, \partial G), d(y, \partial G)\}}\right) .
$$

Definition 1.1. A domain $G \varsubsetneqq \mathbb{R}^{n}$ has the comparison property if there exists a constant $K$ such that $j_{G} / K \leq \alpha_{G} \leq 2 j_{G}$.

The upper bound from the previous definition always holds and the constant 2 is the best possible, as was proved in [2, Theorem 3.2]. Next, we define the exterior ball condition, which played an important role in [10] that dealt with the comparison property. Several related conditions from the literature were reviewed in [10, Section 3].

Definition 1.2. Let $G \varsubsetneqq \mathbb{R}^{n}$ and $L \geq 1$. A domain $G$ is said to satisfy the $L$-exterior ball condition ( $L-E B$ condition) if, for every $x \in \partial G \backslash\{\infty\}$ and $r>0$, there exists a point $z \in \overline{B^{n}}(x, r)$ such that $B^{n}(z, r / L) \subset G^{c}$.

In [10], it was shown that every EB domain has the comparison property. Unfortunately, the constant in that paper was $9 \mathrm{~L}$, whereas we would like to have a constant that tends to 1 as $L \rightarrow 1$, since it is known [14, Theorem 4.2] that this constant equals 1 for 1-EB domains. In fact, we can calculate the optimal constant for every $L \geq 1$.

THEOREM 1.3. If $G \varsubsetneqq \mathbb{R}^{n}$ has the $L-E B$ property, then $G$ has the comparison property with constant $L+\sqrt{L^{2}-1}$. This constant is the best possible one depending only on $L$. 
In Section 5, we consider the Apollonian bilipschitz modulus which was introduced in [10]. For $L \geq 1$ and $G \varsubsetneqq \mathbb{R}^{n}$, we define

$$
\alpha_{L}(G):=\sup _{f} \sup _{x, y \in G}\left\{\frac{\alpha_{f(G)}(f(x), f(y))}{\alpha_{G}(x, y)}, \frac{\alpha_{G}(x, y)}{\alpha_{f(G)}(f(x), f(y))}\right\},
$$

where the first supremum is taken over all $L$-bilipschitz mappings $f: G \rightarrow \mathbb{R}^{n}$ (with the understanding that terms with zero denominators are ignored). Notice that the second supremum is the Apollonian bilipschitz modulus of $f$, that is, the least constant for which $f$ is Apollonian bilipschitz. Hence, $\alpha_{L}(G)<\infty$ if and only if every $L$-bilipschitz mapping is Apollonian bilipschitz as well, with uniformly bounded constant.

The next result answers the question stated in the first paragraph of this paper regarding getting small Apollonian bilipschitz constants.

Theorem 1.4. If $G \varsubsetneqq \mathbb{R}^{n}$ is a domain, then

$$
\lim _{L \rightarrow 1} \alpha_{L}(G)=1
$$

if and only if $G$ is a ball or half-space.

\section{Notation and terminology}

Sections $2.1,2.2$, and 2.3 contain fairly standard material and can be perused by the seasoned reader. Sections 2.4 and 2.5, on the other hand, contain material specific to the Apollonian metric.

2.1. The Möbius space. We denote by $\left\{e_{1}, e_{2}, \ldots, e_{n}\right\}$ the standard basis of $\mathbb{R}^{n}$ and by $n$ the dimension of the Euclidean space under consideration and we assume that $n \geq 2$. For $x \in \mathbb{R}^{n}$, we denote by $x_{i}$ its $i$ th coordinate. The following notation is used for balls, spheres, and the upper half-space ( $x \in \mathbb{R}^{n}$ and $0<r<\infty)$ :

$$
\begin{aligned}
& B^{n}(x, r):=\left\{y \in \mathbb{R}^{n}:|x-y|<r\right\}, \quad S^{n-1}(x, r):=\partial B^{n}(x, r), \\
& B^{n}:=B^{n}(1), \quad S^{n-1}:=S^{n-1}(1), \quad H^{n}:=\left\{y \in \mathbb{R}^{n}: y_{n}>0\right\} .
\end{aligned}
$$

We use the notation $\overline{\mathbb{R}^{n}}:=\mathbb{R}^{n} \cup\{\infty\}$ for the one-point compactification of $\mathbb{R}^{n}$. We define the spherical (chordal) metric $q$ in $\overline{\mathbb{R}^{n}}$ by means of the canonical projection onto the Riemann sphere. We consider $\overline{\mathbb{R}^{n}}$ as the metric space $\left(\overline{\mathbb{R}^{n}}, q\right)$, hence, its balls are the (open) balls of $\mathbb{R}^{n}$, half-spaces, and complements of closed balls. If $G \subset \overline{\mathbb{R}^{n}}$, we denote by $\partial G, G^{c}$, and $\bar{G}$ its boundary, complement, and closure, respectively, all with respect to $\overline{\mathbb{R}^{n}}$. In contrast to topological operations, we consider metric operations with respect to the ordinary Euclidean metric. 
1144 Limits of the comparison and bilipschitz properties

2.2. Möbius mappings. The cross ratio $|a, b, c, d|$ is defined by

$$
|a, b, c, d|:=\frac{q(a, c) q(b, d)}{q(a, b) q(c, d)}\left(=\frac{|a-c||b-d|}{|a-b||c-d|}\right)
$$

for $a \neq b, c \neq d$, and $a, b, c, d \in \overline{\mathbb{R}^{n}}$, where the second equality holds if $a, b, c, d \in$ $\mathbb{R}^{n}$. A homeomorphism $f: \overline{\mathbb{R}^{n}} \rightarrow \overline{\mathbb{R}^{n}}$ is a Möbius mapping if

$$
|f(a), f(b), f(c), f(d)|=|a, b, c, d|
$$

for every quadruple $a, b, c, d \in \overline{\mathbb{R}^{n}}$ with $a \neq b$ and $c \neq d$. For more information on Möbius mappings, see, for example, [1, Section 3]. Using the cross ratio, we can express the Apollonian metric as

$$
\alpha_{G}(x, y)=\sup _{a, b \in \partial G}|a, y, x, b|
$$

for $x, y \in G \subset \overline{\mathbb{R}^{n}}$. This means, in particular, that $\alpha_{G}$ is Möbius invariant, as was noted in [2, Introduction (2)].

2.3. Some miscellaneous notation and terminology. (i) For $x \in G \varsubsetneqq \mathbb{R}^{n}$, we denote $\delta(x):=d(x, \partial G):=\min \{|x-z|: z \in \partial G\}$.

(ii) For $x, y, z \in \mathbb{R}^{n}$, we denote by $\widehat{x y z}$ the smallest angle between the vectors $x-y$ and $z-y$.

(iii) For $x, y \in \mathbb{R}^{n}$, we denote by $x y$ the line through $x$ and $y$ and by $[x, y]$ the closed segment between $x$ and $y$.

2.4. The Apollonian balls approach. The Apollonian balls approach has previously been used in $[2,3]$ and $[14$, Theorem 4.1$]$ although this presentation is from [10, Section 5.1]. The notation of this section will be used practically in every proof in this paper.

For $x, y \in G \varsubsetneqq \mathbb{R}^{n}$, we define

$$
q_{x}:=\sup _{b \in \partial G} \frac{|b-y|}{|b-x|}, \quad q_{y}:=\sup _{a \in \partial G} \frac{|a-x|}{|a-y|} .
$$

The numbers $q_{x}$ and $q_{y}$ are called the Apollonian parameters of $x$ and $y$ (with respect to $G)$. By definition, $\alpha_{G}(x, y)=\log \left(q_{x} q_{y}\right)$. The Apollonian balls are defined by

$$
\begin{aligned}
& B_{x}:=\left\{z \in \mathbb{R}^{n}: \frac{|z-x|}{|z-y|}<\frac{1}{q_{x}}\right\}, \\
& B_{y}:=\left\{z \in \mathbb{R}^{n}: \frac{|z-y|}{|z-x|}<\frac{1}{q_{y}}\right\} .
\end{aligned}
$$


We collect the following immediate results regarding these balls:

(1) $B_{x} \subset G$ and $\overline{B_{x}} \cap \partial G \neq \varnothing$, similarly for $B_{y}$;

(2) if $i_{x}$ and $i_{y}$ denote the inversions in the spheres $\partial B_{x}$ and $\partial B_{y}$, then $y=$ $i_{x}(x)=i_{y}(x)$

(3) since $\infty \notin G$, we have $q_{x}, q_{y} \geq 1$; if $\infty \notin \bar{G}$, then $q_{x}, q_{y}>1$;

(4) if $x_{0}$ denotes the center of $B_{x}$ and $r_{x}$ its radius, then

$$
\left|x-x_{0}\right|=\frac{|x-y|}{q_{x}^{2}-1}=\frac{r_{x}}{q_{x}}
$$

(5) we have $q_{x}-1 \leq|x-y| / \delta(x) \leq q_{x}+1$.

2.5. Quasi-isotropy. We define the concept of quasi-isotropy which is a kind of local comparison property. It was introduced in [11] and was the focus of [9]; however, it was originally conceived of by the author in order to prove Theorem 1.4 on the Apollonian bilipschitz modulus.

This property is the weakest regularity property of the Apollonian metric which we consider. Thus, we will show in Lemma 5.5 that the Apollonian bilipschitz constant $\alpha_{L}(G)$ is always greater than or equal to the quasi-isotropy constant. Similarly, it was shown in [11, Section 4] that if $G$ has the comparison property with constant $K$, then $G$ is $2 K$-quasi-isotropic. This means that the quasi-isotropy constant gives us a lower bound for the comparison constant, a fact that we will use in the proof of Theorem 1.3.

Definition 2.1. A metric space $(G, d)$ with $G \subset \mathbb{R}^{n}$ is $K$-quasi-isotropic if

$$
\limsup _{r \rightarrow 0} \frac{\sup \{d(x, z):|x-z|=r\}}{\inf \{d(x, y):|x-y|=r\}} \leq K
$$

for every $x \in G$. A metric which is 1-quasi-isotropic is said to be isotropic, whereas a metric that is not $K$-quasi-isotropic, for any $K$, is said to be anisotropic. The function $q i$ is defined on the set of domains in $\mathbb{R}^{n}$ so that $q i(G)$ is the least constant for which $\alpha_{G}$ is quasi-isotropic or $q i(G)=\infty$ if $\alpha_{G}$ is anisotropic.

We will only use quasi-isotropy in a very tangential manner in this paper, hence, we will not expose here any methods for calculating the quasi-isotropy constant. For a presentation of such techniques, the reader is referred to [9].

\section{The comparison constant of an exterior ball domain}

In this section, we calculate the exact value of the comparison constant for EB domains. We start with a geometrical lemma which is similar to [9, Lemma 3.6], except that we now consider the Apollonian balls about two points instead of the Apollonian spheres through one point.

Lemma 3.1. Let $x, y \in G \varsubsetneqq \mathbb{R}^{n}$ and let $B_{x}$ and $B_{y}$ be the corresponding Apollonian balls. If $B:=B^{n}(b, r)$ is a ball with $r>d\left(B_{x}, B_{y}\right) / 2$ which does not intersect $B_{x} \cup B_{y}$, 
1146 Limits of the comparison and bilipschitz properties

then

$$
|x-b| \geq \sqrt{r^{2}+\frac{2 r q_{y}|x-y|}{\left(q_{x} q_{y}-1\right)}} .
$$

Proof. We may assume that $B$ is tangent to both $B_{x}$ and $B_{y}$, since otherwise $\mid x-$ $b \mid$ is smaller for some other ball with the same radius, or we can choose another ball with the same distance to $x$ but with a larger $r$.

Denote the centers of $B_{x}$ and $B_{y}$ by $x_{0}$ and $y_{0}$ and set $\theta:=\widehat{y_{0} x_{0} b}, s:=\left|x-x_{0}\right|$, and $w:=\left|x_{0}-y_{0}\right|$. Using the cosine rule in the triangles $y_{0} x_{0} b$ and $x x_{0} b$, we get that

$$
\begin{gathered}
\left(r+r_{y}\right)^{2}=\left(r+r_{x}\right)^{2}+w^{2}-2\left(r+r_{x}\right) w \cos \theta \\
|x-b|^{2}=\left(r+r_{x}\right)^{2}+s^{2}-2\left(r+r_{x}\right) s \cos \theta .
\end{gathered}
$$

Combining these equations to eliminate $\cos \theta$, we get that

$$
|x-b|^{2}=\frac{w-s}{w}\left(r+r_{x}\right)^{2}+\frac{s}{w}\left(r+r_{y}\right)^{2}+s(s-w) .
$$

It follows from the definition of $q_{x}$ and $q_{y}$ (cf. Result (4) in Section 2.4) that $s=|x-y| /\left(q_{x}^{2}-1\right), r_{x}=|x-y| q_{x} /\left(q_{x}^{2}-1\right), r_{y}=|x-y| q_{y} /\left(q_{y}^{2}-1\right)$, and

$$
\begin{aligned}
w & =\left|x_{0}-x\right|+|x-y|+\left|y-y_{0}\right|=\left(\frac{1}{q_{x}^{2}-1}+1+\frac{1}{q_{y}^{2}-1}\right)|x-y| \\
& =\frac{q_{x}^{2} q_{y}^{2}-1}{\left(q_{x}^{2}-1\right)\left(q_{y}^{2}-1\right)}|x-y| .
\end{aligned}
$$

Using these in (3.3), we get that

$$
\begin{aligned}
|x-b|^{2}= & \frac{q_{y}^{2}\left(q_{x}^{2}-1\right)\left(r+r_{x}\right)^{2}}{q_{x}^{2} q_{y}^{2}-1}+\frac{\left(q_{y}^{2}-1\right)\left(r+r_{y}\right)^{2}}{q_{x}^{2} q_{y}^{2}-1}-\frac{|x-y|^{2}}{q_{x}^{2}-1}\left(1+\frac{1}{q_{y}^{2}-1}\right) \\
= & {\left[\left(q_{y}^{2} q_{x}^{2}-1\right) r^{2}+2\left(q_{x} q_{y}+1\right) q_{y} r|x-y|\right.} \\
& \left.+\frac{q_{x}^{2} q_{y}^{4}-q_{y}^{2}}{\left(q_{x}^{2}-1\right)\left(q_{y}^{2}-1\right)}|x-y|^{2}\right] \frac{1}{q_{x}^{2} q_{y}^{2}-1}-\frac{q_{y}^{2}|x-y|^{2}}{\left(q_{x}^{2}-1\right)\left(q_{y}^{2}-1\right)} \\
= & r^{2}+2 \frac{q_{y}|x-y| r}{q_{x} q_{y}-1} .
\end{aligned}
$$

We also need the following lemma which is a variant of [14, Theorem 4.2]. 
Lemma 3.2. Let $x, y \in G \varsubsetneqq \mathbb{R}^{n}$ and let $B_{x}$ and $B_{y}$ be the Apollonian balls. If the convex hull of $B_{x} \cup B_{y}$ does not intersect $\partial G$, then $j_{G}(x, y) \leq \alpha_{G}(x, y)$.

The proof of Theorem 1.3 is quite similar to the proof of [9, Theorem 1.4(1)] in which we estimated the quasi-isotropy constant of an EB domain. However, since there is a gap between the Apollonian balls, it follows that for a large enough $\alpha_{G}(x, y)$, the EB property becomes worthless. Thus, we proceed in two steps this time; first, considering points with small Apollonian distance (the next lemma) and then using an ad hoc measure to take care of the rest of the points in the proof of Theorem 1.3.

Lemma 3.3. Let $G \varsubsetneqq \mathbb{R}^{n}$ be an $L-E B$ domain for $L>1$ and let $x, y \in G$ be points such that

$$
\alpha_{G}(x, y)<\log \left(2 \sqrt{\frac{L+1}{L-1}}-1\right)
$$

Then

$$
\alpha_{G}(x, y) \geq\left(L-\sqrt{L^{2}-1}\right) j_{G}(x, y) .
$$

Proof. Fix $x, y \in G$, satisfying inequality (3.6), let $B_{x}$ and $B_{y}$ be the Apollonian balls, and let $q_{x}$ and $q_{y}$ be the Apollonian ball parameters, as described in Section 2.4 .

If there are no points of $\partial G$ in the convex hull $C$ of $B_{x} \cup B_{y}$, then $\alpha_{G}(x, y) \geq$ $j_{G}(x, y)$ by Lemma 3.2 and there is nothing to prove. We may thus assume that $C \cap \partial G \neq \varnothing$. Fix $r>d\left(B_{x}, B_{y}\right) / 2$. For $\zeta \in C$, let $B$ be the ball with radius $r$, tangent to $B_{x}$ and $B_{y}$ for which the distance $h:=d(\zeta, B)$ is minimal. Then every ball with radius $r$ and center in $B^{n}(\zeta, h+r)$ intersects $G$. This means that if $\zeta$ was a boundary point of $G$, then $G$ would not be EB with constant smaller than $(h+r) / r=|\zeta-b| / r$, where $b$ denotes the center of the ball. Since we know that $G$ is $L$-EB, it follows that $|\zeta-b| \leq L r$ for $\zeta \in C \cap \partial G$.

Combining this with Lemma 3.1, we find that

$$
\begin{aligned}
\delta(x) & =\inf _{\zeta \in C \cap \partial G}|x-b|-|\zeta-b| \geq|x-b|-L r \\
& =\sqrt{r^{2}+\frac{2 r q_{y}|x-y|}{\left(q_{x} q_{y}-1\right)}}-L r=: f(r)
\end{aligned}
$$

for all $r>d\left(B_{x}, B_{y}\right) / 2$. We choose $r$ so as to maximize the lower bound.

We find that $d f / d r=(r+c) / \sqrt{r^{2}+2 r c}-L$, where we denote that $c:=q_{y} \mid x-$ $y \mid /\left(q_{x} q_{y}-1\right)$. Hence, $f$ has a maximum at $r_{0}=c\left(L / \sqrt{L^{2}-1}-1\right)$. We need to check that $r_{0}>d\left(B_{x}, B_{y}\right) / 2$ so that Lemma 3.1 is applicable for this value of $r$. 
1148 Limits of the comparison and bilipschitz properties

The inequality $r_{0}>d\left(B_{x}, B_{y}\right) / 2$ is equivalent to

$$
\begin{aligned}
2\left(\frac{L}{\sqrt{L^{2}-1}}-1\right) \frac{q_{y}|x-y|}{q_{x} q_{y}-1} & >|x-y|-d\left(x, \partial B_{x}\right)-d\left(y, \partial B_{y}\right) \\
& =|x-y|\left(1-\frac{1}{q_{x}+1}-\frac{1}{q_{y}+1}\right) \\
& =|x-y| \frac{q_{x} q_{y}-1}{\left(q_{x}+1\right)\left(q_{y}+1\right)} .
\end{aligned}
$$

We thus have to show that

$$
2\left(\frac{L}{\sqrt{L^{2}-1}}-1\right)>\frac{\left(q_{x} q_{y}-1\right)^{2}}{q_{y}\left(q_{x}+1\right)\left(q_{y}+1\right)} .
$$

The denominator of the right-hand side of this estimate equals $\left(q_{x} q_{y}+q_{y}\right)\left(q_{y}+\right.$ $1)$. Since $q_{y} \geq 1$, we get the lower bound $2\left(q_{x} q_{y}+1\right)$ for this denominator. Since $\exp \alpha_{G}(x, y)=q_{x} q_{y}$, we see that it suffices to show that

$$
4\left(\frac{L}{\sqrt{L^{2}-1}}-1\right)\left(\exp \alpha_{G}(x, y)+1\right)>\left(\exp \alpha_{G}(x, y)-1\right)^{2} .
$$

Solving this second-degree equation in $\exp \alpha_{G}(x, y)$ gives

$$
\exp \alpha_{G}(x, y)<2 \sqrt{\frac{(L+1)}{(L-1)}}-1,
$$

which is the assumption of the lemma.

We then set $r=r_{0}$ in the estimate (3.8), which gives

$$
\frac{|x-y|}{\delta(x)} \leq\left(L+\sqrt{L^{2}-1}\right) \frac{q_{x} q_{y}-1}{q_{x}} .
$$

We can derive a similar estimate for $\delta(y)$ and so we find that

$$
\frac{|x-y|}{\min \{\delta(x), \delta(y)\}} \leq\left(L+\sqrt{L^{2}-1}\right) \frac{q_{x} q_{y}-1}{\min \left\{q_{x}, q_{y}\right\}} .
$$

Using the Bernoulli inequality, we find that

$$
\begin{aligned}
\frac{j_{G}(x, y)}{L+\sqrt{L^{2}-1}} & \leq \log \left(1+\frac{|x-y|}{\left(L+\sqrt{L^{2}-1}\right) \min \{\delta(x), \delta(y)\}}\right) \\
& \leq \log \left(1+\frac{q_{x} q_{y}-1}{\min \left\{q_{x}, q_{y}\right\}}\right) \leq \log \left(q_{x} q_{y}\right)=\alpha_{G}(x, y),
\end{aligned}
$$

which was to be shown. 
Proof of Theorem 1.3. We first note that if $L=1$, then $G$ is convex and the claim follows from [14, Theorem 4.2]. We assume then that $L>1$ and denote $d:=$ $2 \sqrt{(L+1) /(L-1)}-1$. The bound $L+\sqrt{L^{2}-1}$ on the comparison constant holds by Lemma 3.3 if $\alpha_{G}(x, y) \leq \log d$.

Suppose then that $x, y \in G$ are such that $\alpha_{G}(x, y) \geq \log d$. By result (5) in Section 2.4, we always have

$$
\frac{|x-y|}{\min \{\delta(x), \delta(y)\}} \leq \max \left\{1+q_{x}, 1+q_{y}\right\} .
$$

Hence, we find that

$$
\frac{j_{G}(x, y)}{\alpha_{G}(x, y)} \leq \frac{\log \left(2+\max \left\{q_{x}, q_{y}\right\}\right)}{\log \left(q_{x} q_{y}\right)} \leq \frac{\log \left(2+q_{x} q_{y}\right)}{\log \left(q_{x} q_{y}\right)} \leq \frac{\log (2+d)}{\log d} .
$$

The last inequality follows since the function $z \mapsto \log (2+z) / \log z$ is decreasing.

Thus, we have seen that for some points, the ratio $j_{G} / \alpha_{G}$ is bounded from above by $L+\sqrt{L^{2}-1}$ and for all others by $\log (2+d) / \log d$. This means that $G$ has the comparison property with constant less than or equal to

$$
\max \left\{L+\sqrt{L^{2}-1}, \frac{\log (2 \sqrt{(L+1) /(L-1)}+1)}{\log (2 \sqrt{(L+1) /(L-1)}-1)}\right\} .
$$

Next, we prove that the first term in the maximum is always greater than the second one. We introduce a new variable, $u^{2}:=(L+1) /(L-1)$, which satisfies $u>1$.

We have to prove that

$$
\frac{\log (2 u+1)}{\log (2 u-1)} \leq \frac{u^{2}+1}{u^{2}-1}+\left[\left(\frac{u^{2}+1}{u^{2}-1}\right)^{2}-1\right]^{1 / 2}=\frac{u+1}{u-1}
$$

Since $\log (2 u-1)>0$, this is equivalent to

$$
g(u):=\frac{u+1}{u-1} \log (2 u-1)-\log (2 u+1) \geq 0 .
$$

We will show that $g$ is decreasing; we differentiate $g$ :

$$
\frac{d g}{d u}=-\frac{2}{(u-1)^{2}} \log (2 u-1)+\frac{u+1}{u-1} \frac{2}{2 u-1}-\frac{2}{2 u+1} .
$$

Then we multiply the inequality $d g / d u \leq 0$ by $-(u-1)^{2} / 2$ to get the equivalent inequality

$$
\begin{aligned}
h(u) & :=\log (2 u-1)+\frac{(u-1)^{2}}{2 u+1}-\frac{u^{2}-1}{2 u-1} \\
& =\log (2 u-1)-6 u \frac{u-1}{4 u^{2}-1} \geq 0
\end{aligned}
$$


We find that

$$
\frac{d h}{d u}=\frac{2}{2 u-1}-6 \frac{4 u^{2}-2 u+1}{\left(4 u^{2}-1\right)^{2}}=8 \frac{(u-1)\left(2 u^{2}+1\right)}{\left(4 u^{2}-1\right)^{2}}
$$

and so it is clear that $h$ is increasing. It follows that $h(u) \geq h(1)=0$, which means that $d g / d u \leq 0$. Since $g$ is decreasing, it follows that

$$
g(u) \geq \lim _{u \rightarrow \infty} g(u) \geq \lim _{u \rightarrow \infty} \log (2 u-1)-\log (2 u+1)=0
$$

which means that the first term in the maximum is greater than the second one and completes the proof that $L+\sqrt{L^{2}-1}$ is an upper bound for the comparison constant.

To show that this constant is the best possible, recall from Section 2.5 that the quasi-isotropy constant is always less than one half of the comparison constant. It was proven in $[9$, Theorem $1.4(1)]$ that there exists an $L$-EB domain with quasi-isotropy constant $2\left(L+\sqrt{L^{2}-1}\right)$ and so the comparison constant of this domain is at least $L+\sqrt{L^{2}-1}$, which concludes the proof.

\section{The spiral mapping}

In this section, we will define a bilipschitz mapping that has large rotational distortion even with small bilipschitz constant. This mapping is a generalization of a mapping of $\mathbb{R}^{2}$ onto itself considered by Freedman and He in [4]. Note that the difficulty in extending it to $\mathbb{R}^{n}$ lies therein, that we wish to preserve the property $f(x)=x$ for $x \notin B^{n}$. The following quite lengthy proof is based on a series of elementary estimates.

Lemma 4.1. Let $P \geq 1$ and let $x=(r \cos \theta, r \sin \theta, \hat{x}) \in \mathbb{R}^{n}$, where $r \in[0, \infty), \theta \in$ $[0,2 \pi)$, and $\hat{x} \in \mathbb{R}^{n-2}$. Let $\theta^{\prime}:=\theta+(P-1 / P) \log (r /(1-|\hat{x}|))$. The mapping $f: \mathbb{R}^{n} \rightarrow \mathbb{R}^{n}$ given by

$$
f(x)=f(r \cos \theta, r \sin \theta, \hat{x}):=\left(r \cos \theta^{\prime}, r \sin \theta^{\prime}, \hat{x}\right)
$$

for $r+|\hat{x}|<1$ and $f(x)=x$ for $r+|\hat{x}| \geq 1$ is $P^{2}$-bilipschitz.

Proof. Suppose that $x$ and $y$ are two points in $\mathbb{R}^{n}$ with $x=(r \cos \theta, r \sin \theta, \hat{x})$ and $y=(s \cos \phi, s \sin \phi, \hat{y})$ where $\hat{x}, \hat{y} \in \mathbb{R}^{n-2}$. We define

$$
H:=\left\{z \in \mathbb{R}^{n}: \sqrt{z_{1}^{2}+z_{2}^{2}}+\sqrt{z_{3}^{2}+\cdots+z_{n}^{2}}<1\right\}
$$

Observe that $H$ is precisely the domain in which $f$ is not by definition equal to the identity. We first assume that $r+|\hat{x}|<1$ and that $s+|\hat{y}|<1$, that is, $x, y \in H$. 
We need to show that

$$
\begin{aligned}
P^{4}|x-y|^{2} & =P^{4}\left[(r \cos \theta-s \cos \phi)^{2}+(r \sin \theta-s \sin \phi)^{2}+|\hat{x}-\hat{y}|^{2}\right] \\
& \geq\left(r \cos \theta^{\prime}-s \cos \phi^{\prime}\right)^{2}+\left(r \sin \theta^{\prime}-s \sin \phi^{\prime}\right)^{2}+|\hat{x}-\hat{y}|^{2} \\
& =|f(x)-f(y)|^{2},
\end{aligned}
$$

where

$$
\begin{aligned}
& \theta^{\prime}:=\theta+\left(P-\frac{1}{P}\right) \log \left(\frac{r}{1-|\hat{x}|}\right), \\
& \phi^{\prime}:=\phi+\left(P-\frac{1}{P}\right) \log \left(\frac{s}{1-|\hat{y}|}\right) .
\end{aligned}
$$

This inequality can be reexpressed as

$$
\left(P^{4}-1\right)\left[r^{2}+s^{2}+|\hat{x}-\hat{y}|^{2}\right] \geq 2 r s\left[P^{4} \cos \gamma-\cos (\gamma+\lambda)\right],
$$

where $\gamma:=\theta-\phi$ and $\lambda:=(P-1 / P)[\log (r /(1-|\hat{x}|))-\log (s /(1-|\hat{y}|))]$. We will use the elementary estimate

$$
\begin{aligned}
& P^{4} \cos \gamma-\cos (\gamma+\lambda) \\
& \quad=\left(P^{4}-\cos \lambda\right) \cos \gamma+\sin \lambda \sin \gamma \leq \sqrt{\left(P^{4}-\cos \lambda\right)^{2}+\sin ^{2} \lambda} \\
& \quad=\sqrt{P^{8}+1-2 P^{4} \cos \lambda} \leq \sqrt{P^{8}+1-2 P^{4}+2 P^{4} \lambda^{2}} \\
& \quad=\left(P^{2}-1\right) \sqrt{\left(1+P^{2}\right)^{2}+2 P^{2}\left[\log \left(\frac{r}{1-|\hat{x}|}\right)-\log \left(\frac{s}{1-|\hat{y}|}\right)\right]^{2}} .
\end{aligned}
$$

We use (4.6) in (4.5) and see that it is sufficient to prove that

$$
\begin{aligned}
& \left(P^{2}+1\right)\left[r^{2}+s^{2}+|\hat{x}-\hat{y}|^{2}\right] \\
& \quad \geq\left(P^{2}+1\right)\left[r^{2}+s^{2}+(|\hat{x}|-|\hat{y}|)^{2}\right] \\
& \quad \geq 2 r s \sqrt{\left(P^{2}+1\right)^{2}+2 P^{2}\left[\log \left(\frac{r}{s}\right)+\log \left(\frac{1-|\hat{y}|}{1-|\hat{x}|}\right)\right]^{2}} .
\end{aligned}
$$

We divide through by $\left(P^{2}+1\right)$. Since $2 P^{2} /\left(P^{2}+1\right)^{2} \leq 1 / 2$, it suffices to prove that

$$
\frac{\left[r^{2}+s^{2}+(|\hat{x}|-|\hat{y}|)^{2}\right]^{2}}{(2 r s)^{2}} \geq 1+\left(\frac{1}{2}\right)\left[\log \left(\frac{r}{s}\right)+\log \left(\frac{1-|\hat{y}|}{1-|\hat{x}|}\right)\right]^{2} .
$$

We assume, without loss of generality, that $r \geq s$ and denote that $|\hat{x}|-|\hat{y}|=c$. We see that $(1-|\hat{y}|) /(1-|\hat{x}|)=1+c /(1-|\hat{x}|)$ is maximized by maximizing $|\hat{x}|$ for $c>0$, and that the ratio is smaller than 1 for $c<0$. Since $|\hat{x}|<1-r$, we see 
1152 Limits of the comparison and bilipschitz properties

that the right-hand side is less than or equal to

$$
1+\frac{[\log (r s)+\log ((r+c) / r)]^{2}}{2}=1+\frac{\log ^{2}((r+c) s)}{2} .
$$

We introduce the variables $u:=r / s \geq 1$ and $v:=c^{2} /(r s)$. Then we have to prove that

$$
\begin{aligned}
\frac{\left[r^{2}+s^{2}+c^{2}\right]^{2}}{(2 r s)^{2}} & =\frac{[u+1 / u+v]^{2}}{4} \geq 1+\frac{\log ^{2}(u+\sqrt{u v})}{2} \\
& =1+\frac{\log ^{2}((r+c) / s)}{2} .
\end{aligned}
$$

We define yet another variable $w:=u+\sqrt{u v}$. We will consider how $u+1 / u+v$ varies for fixed $w \geq 1$. Since $v=(w-u)^{2} / u$, this amounts to considering the function

$$
g(u):=u+\frac{1}{u}+\frac{(w-u)^{2}}{u}=2 u-2 w+\frac{1+w^{2}}{u} .
$$

Now, $g^{\prime}(u)=2-\left(1+w^{2}\right) / u^{2}$ has one zero at $u=\sqrt{\left(1+w^{2}\right) / 2}$, which is a minimum of $g$. Hence,

$$
g(u) \geq \sqrt{2\left(1+w^{2}\right)}-2 w+\frac{1+w^{2}}{\sqrt{\left(1+w^{2}\right) / 2}}=2 \sqrt{2} \sqrt{1+w^{2}}-2 w,
$$

and we see that it suffices to prove that

$$
\begin{aligned}
\frac{\left[2 \sqrt{2} \sqrt{1+w^{2}}-2 w\right]^{2}}{4} & =\left[\sqrt{2} \sqrt{1+w^{2}}-w\right]^{2} \\
& =2+3 w^{2}-w \sqrt{8\left(1+w^{2}\right)} \geq 1+\frac{\log ^{2}(w)}{2}
\end{aligned}
$$

for $w \geq 1$. Clearly, this inequality holds for $w=1$ and so it suffices to show that the left-hand side grows faster than the right-hand side. In terms of derivatives, this means that

$$
6 w-\sqrt{8} \frac{1+2 w^{2}}{\sqrt{1+w^{2}}} \geq \frac{\log w}{w}
$$

Since $\log w \leq w-1$, it suffices to show that

$$
\left(6 w^{2}-w+1\right) \sqrt{1+w^{2}} \geq \sqrt{8}\left(2 w^{2}+1\right) w
$$


Squaring both sides and collecting all terms on one side, we see that this inequality is equivalent to

$$
\left[(2 w-1)^{2} w^{2}+4 w^{2}+1\right](w-1)^{2} \geq 0
$$

which is obvious. We have now proved that $f$ is $P^{2}$-lipschitz in $H$.

Next, assume that $x \in H$ and $y \notin H$. Let $z$ be the point on $\partial H$ such that $\mid x-$ $z|+| z-y|=| x-y \mid$. Since $f(z)=z$ and $f(y)=y$, we have

$$
\begin{aligned}
|f(x)-f(y)| & \leq|f(x)-f(z)|+|f(z)-f(y)| \\
& \leq P^{2}|x-z|+|z-y| \leq P^{2}|x-y| .
\end{aligned}
$$

Finally, the case $x, y \notin H$ is trivial since $f$ is the identity for these points.

The inverse of $f$ is of the same form as $f$; only the direction of rotation is changed. It is therefore clear that $f^{-1}$ is $P^{2}$-lipschitz too, and so $f$ is $P^{2}$ bilipschitz.

\section{The limiting behavior of the Apollonian bilipschitz modulus}

In this section, we study how the quantity $\alpha_{L}(G)$ behaves when $L \rightarrow 1$. The results derived on the behavior of $\alpha_{L}(G)$ in [10] are useful only for large $L$ and thus these two approaches are complementary. We prove in Theorem 1.4 that $\alpha_{L}(G) \rightarrow 1$ as $L \rightarrow 1$ if and only if $G$ is a ball or half-space. To prove this result, we need to show two things: if $G$ is a ball or half-space, then $\alpha_{L}(G) \rightarrow 1$ and if $\alpha_{L}(G) \rightarrow 1$, then $G$ is a ball or half-space. The comparison results that have been derived so far in this paper are good for a lower bound on $\alpha_{G}$ and the following result provides the upper bound. This result will suffice for the first implication.

The next lemma uses Seittenranta's metric $\delta_{G}$ in an intermediate step; since this is the only use for the metric here, the reader is referred to [14] for the definition.

Lemma 5.1. Let $f: H^{n} \rightarrow \mathbb{R}^{n}$ be L-bilipschitz and denote $G:=f\left(H^{n}\right)$. Then $f$ is $L^{4}$-lipschitz with respect to the Apollonian metric, that is,

$$
\alpha_{G}(f(x), f(y)) \leq L^{4} \alpha_{H^{n}}(x, y) .
$$

Proof. By [14, Theorem 3.11], we know that the inequality $\alpha_{G} \leq \delta_{G}$ is valid in every domain $G \varsubsetneqq \mathbb{R}^{n}$. Also, we have $\alpha_{H^{n}}=\delta_{H^{n}}$ since both metrics are equal to the hyperbolic metric in the half-space (by [2, Lemma 3.1] and [17, Lemma 8.39] for $\alpha_{H^{n}}$ and $\delta_{H^{n}}$, respectively). It follows that

$$
\alpha_{G}(f(x), f(y)) \leq \delta_{G}(f(x), f(y)) \leq L^{4} \delta_{H^{n}}(x, y)=L^{4} \alpha_{H^{n}}(x, y),
$$

where the second inequality is stated in [14, Theorem 3.18]. 
As usual, the lower bound for the Apollonian metric is harder to come by. We need a series of lemmas. The next lemma follows easily from an extension result of Väisälä [15].

Lemma 5.2. Let $f: B^{n} \rightarrow \mathbb{R}^{n}$ be an L-bilipschitz mapping. There exists an $L_{0}>1$ such that $f\left(B^{n}\right)$ has the $K(L)$-EB property for $L<L_{0}$ with $K(L) \rightarrow 1$ as $L \rightarrow 1$.

Proof. It follows from [15, Example 6.13] that there exists an $L^{\prime}$-bilipschitz mapping $f^{\prime}: \mathbb{R}^{n} \rightarrow \mathbb{R}^{n}$ such that $\left.f^{\prime}\right|_{B^{n}}=f$. Moreover, $L^{\prime} \rightarrow 1$ as $L \rightarrow 1$. Using this extended mapping, it is easy to see that the claim of the lemma holds.

We need the previous result in the half-space. The following lemma will be used to transfer it to this setting.

Lemma 5.3. Let $G \varsubsetneqq \mathbb{R}^{n}$ and let $f: G \rightarrow \mathbb{R}^{n}$ be an L-bilipschitz mapping. Let $\pi$ be the inversion in a sphere with radius $r>0$ whose center is not in $G$. Then $\pi \circ f \circ \pi$ is $L^{3}$-bilipschitz in $\pi(G)$.

Proof. Denote the center of inversion by $w$. It is well known, and follows easily from the definition of an inversion, that

$$
|\pi(x)-\pi(y)|=\frac{r^{2}|x-y|}{|x-w||y-w|}
$$

for $x, y \in G$. We denote that $x^{\prime}:=\pi(x)$ and $y^{\prime}:=\pi(y)$. It follows from the inequality

$$
\begin{aligned}
|g(x)-g(y)| & =\frac{r^{2}\left|f\left(x^{\prime}\right)-f\left(y^{\prime}\right)\right|}{\left|f\left(x^{\prime}\right)-w\right|\left|f\left(y^{\prime}\right)-w\right|} \\
& \leq \frac{r^{2} L\left|x^{\prime}-y^{\prime}\right|}{\left|x^{\prime}-w\right|\left|y^{\prime}-w\right| / L^{2}}=L^{3}|x-y|,
\end{aligned}
$$

(and similarly for the lower bound) that $g$ is bilipschitz in $\pi(G)$ with constant $L^{3}$. In this inequality, we used $\left|f\left(x^{\prime}\right)+e_{n}\right|=\left|f\left(x^{\prime}\right)+f\left(e_{n}\right)\right| \geq\left|x^{\prime}+e_{n}\right| / L$ and so forth.

Corollary 5.4. Let $f: H^{n} \rightarrow \mathbb{R}^{n}$ be an L-bilipschitz mapping. If $L<L_{0}$, then $f\left(H^{n}\right)$ has the $K(L)$-EB property with $K(L) \rightarrow 1$ as $L \rightarrow 1$.

Proof. Let $\pi$ be the inversion in $S^{n-1}\left(-e_{n}, \sqrt{2}\right)$. Then $\pi \circ f \circ \pi$ satisfies the assumptions of Lemma 5.2 and is thus extendable. If $\tilde{g}$ denotes the extension, we define $\tilde{f}=\pi \circ \tilde{g} \circ \pi$. By Lemma 5.3, this mapping is a bilipschitz extension of $f$. Using this extension, we easily see that the claim holds.

To prove the converse implication of the main theorem, we use the concept of quasi-isotropy. The idea behind the next lemma is that the bilipschitz condition does not constrain rotation very much, provided that it happens in a sufficiently small ball. 
Lemma 5.5. If $G \varsubsetneqq \mathbb{R}^{n}$, then $\alpha_{L}(G) \geq q i(G)$ for every $L>1$.

Proof. Let $G \varsubsetneqq \mathbb{R}^{n}$ be a domain with $1<q i(G)<\infty$ (the claim is trivial if $q i(G)=$ 1 , the case $q i(G)=\infty$ is considered below). Fix $\epsilon>0$ and let $\theta, \phi \in S^{n-1}, x \in G$, and $0<\delta \leq \delta(x) / 2$ be such that $\alpha_{G}(x, x+t \theta) / \alpha(x, x+t \phi)>q i(G)-\epsilon$ for $|t| \leq \delta$.

We know from Section 4 (from [4] for $n=2$ ) that there exists an $L$-bilipschitz mapping $f: \mathbb{R}^{n} \rightarrow \mathbb{R}^{n}$ such that $f(x)=x, f\left(x+\delta^{\prime \prime} \phi\right)=x+\delta^{\prime \prime} \theta$, and $f(z)=z$ for $|x-z|>\delta^{\prime}$, where $0<\delta^{\prime \prime}<\delta^{\prime}<\delta$ and $\delta^{\prime \prime}$ depends on $L$ and $\delta^{\prime}$. Note that for this mapping, we have $f(G)=G$. It follows that

$$
\alpha_{L}(G) \geq \frac{\alpha_{f(G)}\left(f(x), f\left(x+\delta^{\prime \prime} \phi\right)\right)}{\alpha_{G}\left(x, x+\delta^{\prime \prime} \phi\right)}=\frac{\alpha_{G}\left(x, x+\delta^{\prime \prime} \theta\right)}{\alpha_{G}\left(x, x+\delta^{\prime \prime} \phi\right)}>q i(G)-\epsilon .
$$

Since $\epsilon>0$ is arbitrary, it follows that $\alpha_{L}(G) \geq q i(G)$.

If $q i(G)=\infty$, then for every $\epsilon>0$, we find $\theta, \phi \in S^{n-1}, x \in G$, and $0<\delta \leq$ $\delta(x) / 2$ such that $\alpha_{G}(x, x+t \theta) / \alpha(x+t \phi)>1 / \epsilon$ for $|t| \leq \delta$. We then argue as above that $\alpha_{L}(G)>1 / \epsilon$, and so we find that $\alpha_{L}(G)=\infty$.

We are now ready for the proof of the main result.

Proof of Theorem 1.4. Suppose first that $\alpha_{L}(G) \rightarrow 1$ as $L \rightarrow 1$. It follows from Lemma 5.5 that $q i(G) \leq 1$, hence, $q i(G)=1$, that is, $G$ is isotropic. It then follows from [9, Theorem 1.10] that $G$ is a ball or half-space.

Next, suppose that $G$ is a half-space and assume, without loss of generality, that $G=H^{n}$. Let $f: H^{n} \rightarrow \mathbb{R}^{n}$ be an $L$-bilipschitz mapping. Then by Lemma 5.1 we have $\alpha_{f\left(H^{n}\right)} \leq L^{4} \alpha_{H^{n}}$ and so only the lower bound remains to be established (the situation is asymmetric, since $H^{n}$ is a half-space, but $f\left(H^{n}\right)$ need not be).

It follows from Corollary 5.4 that $f\left(H^{n}\right)$ is an $L^{\prime}$-EB domain. Hence, it follows from Theorem 1.3 that

$$
\begin{aligned}
\alpha_{f\left(H^{n}\right)}(f(x), f(y)) & \geq\left(L^{\prime}-\sqrt{L^{\prime 2}-1}\right) j_{f\left(H^{n}\right)}(f(x), f(y)) \\
& \geq\left(1-\sqrt{1-L^{\prime-2}}\right) j_{H^{n}}(x, y)
\end{aligned}
$$

since $f$ is $L^{\prime}$-bilipschitz with respect to $j_{H^{n}}$, which follows directly from the Bernoulli inequalities. We see that it suffices to find a lower bound for $j_{H^{n}}(x, y) /$ $\alpha_{H^{n}}(x, y)$.

In the half-space, we have an explicit formula for $\alpha_{H^{n}}$, which equals the hyperbolic metric in this case [1, page 35], namely,

$$
\begin{aligned}
\alpha_{H^{n}}(x, y) & =\operatorname{arcosh}\left(1+\frac{|x-y|^{2}}{2 x_{n} y_{n}}\right) \\
& =\log \left(1+\frac{|x-y|^{2}}{2 x_{n} y_{n}}+\frac{|x-y|}{2 x_{n} y_{n}} \sqrt{|x-y|^{2}+2 x_{n} y_{n}}\right),
\end{aligned}
$$


where $x_{n}$ denotes the $n$th coordinate of $x$. We use the inequality $\log (1+a) /$ $\log (1+b) \geq a / b$, which is valid for $a \leq b$, to estimate $j_{H^{n}} / \alpha_{H^{n}}$. We find that

$$
\begin{aligned}
\frac{j_{H^{n}}(x, y)}{\alpha_{H^{n}}(x, y)} & \geq \frac{|x-y| / \min \left\{x_{n}, y_{n}\right\}}{|x-y|^{2} /\left(2 x_{n} y_{n}\right)+|x-y| \sqrt{|x-y|^{2}+4 x_{n} y_{n}} /\left(2 x_{n} y_{n}\right)} \\
& =\frac{2 x_{n} y_{n}}{\min \left\{x_{n}, y_{n}\right\}} \frac{1}{|x-y|+\sqrt{|x-y|^{2}+4 x_{n} y_{n}}} \\
& \geq \frac{x_{n} y_{n}}{\min \left\{x_{n}, y_{n}\right\}} \frac{1}{|x-y|+\sqrt{x_{n} y_{n}}} \\
& \geq \frac{\sqrt{x_{n} y_{n}}}{|x-y|+\sqrt{x_{n} y_{n}}} \geq \frac{1}{1+|x-y| / \min \left\{x_{n}, y_{n}\right\}},
\end{aligned}
$$

where we used $\min \left\{x_{n}, y_{n}\right\} \leq \sqrt{x_{n} y_{n}}$ in the last two estimates.

Combining this estimate with inequality (5.6), we find that

$$
\begin{aligned}
\alpha_{f\left(H^{n}\right)}(f(x), f(y)) & \geq\left(1-\sqrt{1-L^{\prime-2}}\right) j_{H^{n}}(x, y) \\
& \geq\left(1-\sqrt{1-L^{\prime-2}}\right) \frac{\alpha_{H^{n}}(x, y)}{1+|x-y| / \min \left\{x_{n}, y_{n}\right\}} .
\end{aligned}
$$

This estimate is good only when $|x-y| / \min \left\{x_{n}, y_{n}\right\}$ is small. Thus, we need another approach when $|x-y| / \min \left\{x_{n}, y_{n}\right\}$ is large.

We always have $\alpha_{f\left(H^{n}\right)}(f(x), f(y)) \geq \alpha_{H^{n}}(x, y)-4 \log L$, directly from the definition of the Apollonian metric. Hence, we find that if $\alpha_{H^{n}}(x, y) \geq 4 \log (L) /$ $\sqrt{L-1}$, then

$$
\frac{\alpha_{f\left(H^{n}\right)}(f(x), f(y))}{\alpha_{H^{n}}(x, y)} \geq 1-\frac{4 \log L}{\alpha_{H^{n}}(x, y)} \geq 1-\sqrt{L-1}
$$

On the other hand, if $\alpha_{H^{n}}(x, y) \leq 4 \log (L) / \sqrt{L-1}$, then we have (the first inequality follows from [14, Theorem 4.2] since $H^{n}$ is convex)

$$
\begin{aligned}
1+\frac{|x-y|}{\min \left\{x_{n}, y_{n}\right\}} & =\exp \left\{j_{H^{n}}(x, y)\right\} \\
& \leq \exp \left\{\alpha_{H^{n}}(x, y)\right\} \leq \exp \left\{\frac{4 \log (L)}{\sqrt{L-1}}\right\}
\end{aligned}
$$

We combine the estimates from (5.9) and (5.10). This gives

$$
\frac{\alpha_{f\left(H^{n}\right)}(f(x), f(y))}{\alpha_{H^{n}}(x, y)} \geq \min \left\{\left(1-\sqrt{1-L^{\prime-2}}\right) L^{-4 / \sqrt{L-1}}, 1-\sqrt{L-1}\right\},
$$

irrespective of the value of $\alpha_{H^{n}}(x, y)$. Thus, we have a lower bound that approaches 1 as $L \rightarrow 1$. 
Now, we are done with the case when $H^{n}$ equals a half-space. But using Lemma 5.3, we easily deduce the conclusion for $B^{n}$ from this.

\section{Acknowledgment}

The author wishes to thank Matti Vuorinen for introducing him to the Apollonian metric and for the numerous suggestions on this paper and the other ones in this series. He also thanks Pentti Järvi for pointing out a significant shortcoming in a very early version of this paper. This work was supported in part by the Finnish Academy of Science and Letters and the Academy of Finland.

\section{References}

[1] A. F. Beardon, The Geometry of Discrete Groups, Graduate Texts in Mathematics, vol. 91, Springer-Verlag, New York, 1995.

[2] The Apollonian metric of a domain in $\mathbf{R}^{n}$, Quasiconformal Mappings and Analysis (Ann Arbor, Mich, 1995) (P. Duren, J. Heinonen, B. Osgood, and B. Palka, eds.), Springer-Verlag, New York, 1998, pp. 91-108.

[3] W.-G. Boskoff, Hyperbolic Geometry and Barbilian Spaces, Istituto per la Ricerca di Base. Series of Monographs in Advanced Mathematics, Hadronic Press, Florida, 1996.

[4] M. H. Freedman and Z.-X. He, Factoring the logarithmic spiral, Invent. Math. 92 (1988), no. 1, 129-138.

[5] F. W. Gehring and K. Hag, Hyperbolic geometry and disks, J. Comput. Appl. Math. 105 (1999), no. 1-2, 275-284.

[6] The Apollonian metric and quasiconformal mappings, In the Tradition of Ahlfors and Bers (Stony Brook, NY, 1998) (I. Kra and B. Maskit, eds.), Contemp. Math., vol. 256, Amer. Math. Soc., Rhode Island, 2000, pp. 143-163.

[7] F. W. Gehring and B. G. Osgood, Uniform domains and the quasihyperbolic metric, J. Analyse Math. 36 (1979), 50-74.

[8] F. W. Gehring and B. P. Palka, Quasiconformally homogeneous domains, J. Analyse Math. 30 (1976), 172-199.

[9] P. A. Hästö, The Apollonian metric: quasi-isotropy and Seittenranta's metric, 2003, preprint, http://www.helsinki.fi/ hasto/pp/.

[10] The Apollonian metric: geometry of comparison and bilipschitz domains, Ph.D. thesis (part 1), University of Helsinki, 2003, preprint, http://www.helsinki. fi/ hasto/pp/.

[11] The Apollonian metric: uniformity and quasiconvexity, Ann. Acad. Sci. Fenn. Ser. A I Math. 28 (2003), 385-414.

[12] Z. Ibragimov, The Apollonian metric, sets of constant width and Möbius modulus of ring domains, Ph.D. thesis, University of Michigan, Ann Arbor, Michigan, 2002.

[13] A. D. Rhodes, An upper bound for the hyperbolic metric of a convex domain, Bull. London Math. Soc. 29 (1997), no. 5, 592-594.

[14] P. Seittenranta, Möbius-invariant metrics, Math. Proc. Cambridge Philos. Soc. 125 (1999), no. 3, 511-533.

[15] J. Väisälä, Bi-Lipschitz and quasisymmetric extension properties, Ann. Acad. Sci. Fenn. Ser. A I Math. 11 (1986), no. 2, 239-274. 
1158 Limits of the comparison and bilipschitz properties

[16] M. Vuorinen, Conformal invariants and quasiregular mappings, J. Analyse Math. 45 (1985), 69-115.

[17] _ Conformal Geometry and Quasiregular Mappings, Lecture Notes in Mathematics, vol. 1319, Springer-Verlag, Berlin, 1988.

Peter A. Hästö: Department of Mathematics, University of Michigan, East Hall, Ann Arbor, MI 48109-1109, USA

E-mail address: peter.hasto@helsinki.fi 


\section{Differential Equations \& Nonlinear Mechanics}

\section{An Open Access Journal}

\section{Editor-in-Chief}

K. Vajravelu

USA

Associate Editors

N. Bellomo

Italy

J. L. Bona

USA

J. R. Cannon

USA

S.-N. Chow

USA

B. S. Dandapat

India

E. DiBenedetto

USA

R. Finn

USA

R. L. Fosdick

USA

J. Frehse

Germany

A. Friedman

USA

R. Grimshaw

UK

J. Malek

Czech Republic

J. T. Oden

USA

R. Quintanilla

Spain

K. R. Rajagopal

USA

G. Saccomandi

Italy

Y. Shibata

Japan

Ivar Stakgold

USA

Swaroop Darbha

USA

A. Tani

Japan

S. Turek

Germany

A. Wineman

USA
Website: http://www.hindawi.com/journals/denm/

Aims and Scope

Differential equations play a central role in describing natural phenomena as well as the complex processes that arise from science and technology. Differential Equations \& Nonlinear Mechanics (DENM) will provide a forum for the modeling and analysis of nonlinear phenomena. One of the principal aims of the journal is to promote cross-fertilization between the various subdisciplines of the sciences: physics, chemistry, and biology, as well as various branches of engineering and the medical sciences.

Special efforts will be made to process the papers in a speedy and fair fashion to simultaneously ensure quality and timely publication.

DENM will publish original research papers that are devoted to modeling, analysis, and computational techniques. In addition to original full-length papers, DENM will also publish authoritative and informative review articles devoted to various aspects of ordinary and partial differential equations and their applications to sciences, engineering, and medicine.

\section{Open Access Support}

The Open Access movement is a relatively recent development in academic publishing. It proposes a new business model for academic publishing that enables immediate, worldwide, barrier-free, open access to the full text of research articles for the best interests of the scientific community. All interested readers can read, download, and/or print any Open Access articles without requiring a subscription to the journal in which these articles are published.

In this Open Access model, the publication cost should be covered by the author's institution or research funds. These Open Access charges replace subscription charges and allow the publishers to give the published material away for free to all interested online visitors.

\section{Instructions for Authors}

Original articles are invited and should be submitted through the DENM manuscript tracking system at http://www.mstracking.com/ denm/. Only pdf files are accepted. If, for some reason, submission through the manuscript tracking system is not possible, you can contact denm.support@hindawi.com.

Hindawi Publishing Corporation

410 Park Avenue, 15th Floor, \#287 pmb, New York, NY 10022, USA
HINDAWI 


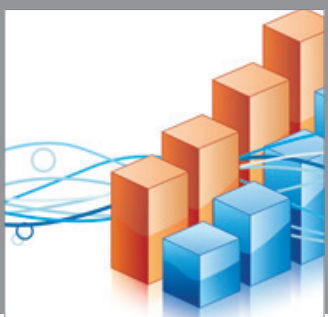

Advances in

Operations Research

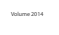

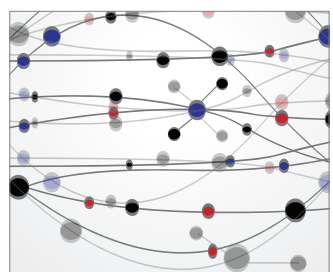

\section{The Scientific} World Journal
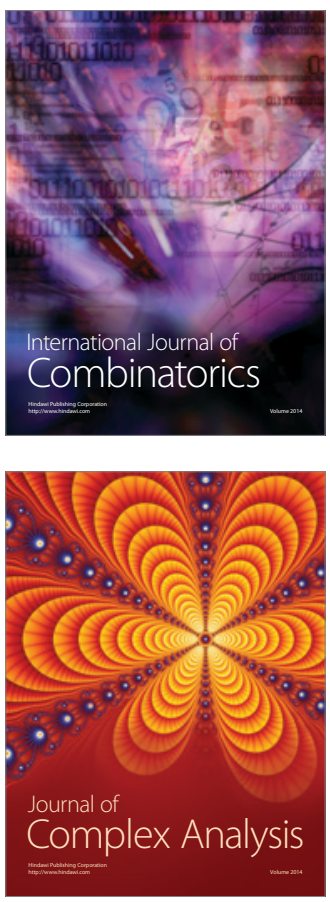

International Journal of

Mathematics and

Mathematical

Sciences
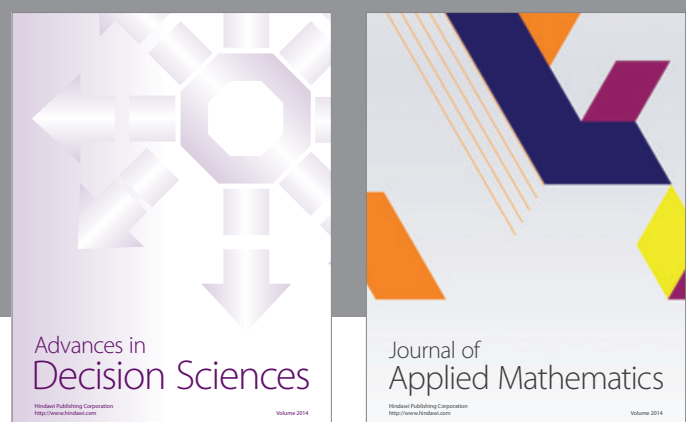

Journal of

Applied Mathematics
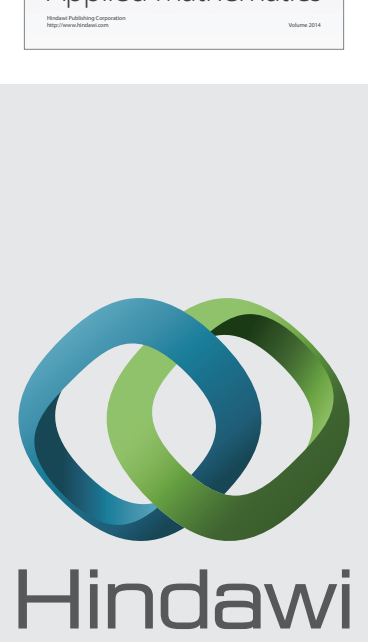

Submit your manuscripts at http://www.hindawi.com
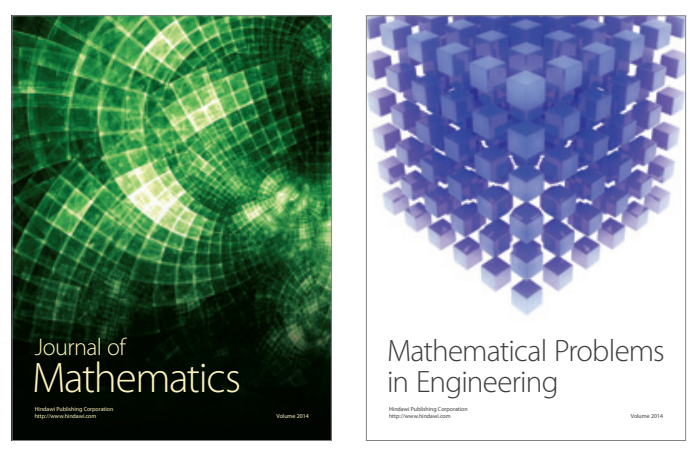

Mathematical Problems in Engineering
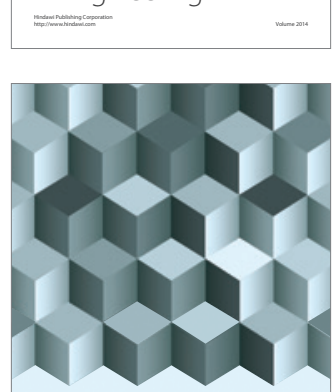

Journal of

Function Spaces
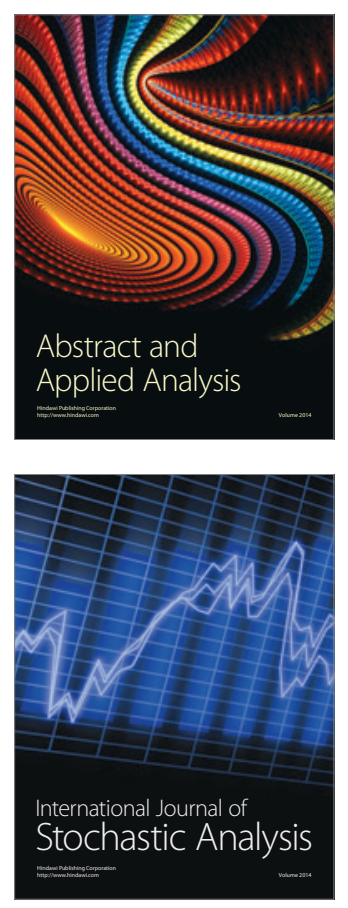

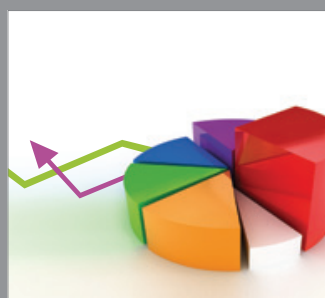

ournal of

Probability and Statistics

Promensencen
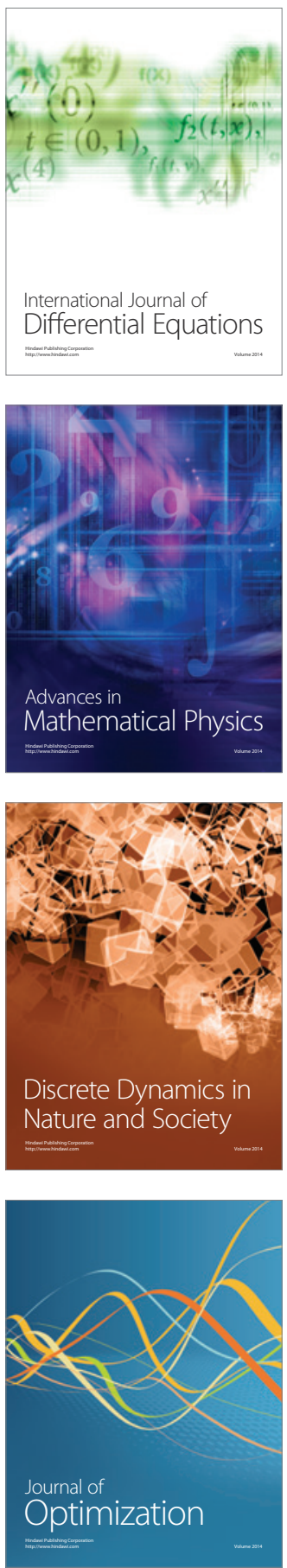